\title{
An overview of exotic plant pest and disease surveillance 2011-2014
}

\author{
M.S. Bullians \\ Ministry for Primary Industries, PO Box 2095, Auckland \\ Corresponding author: mark.bullians@mpi.govt.nz
}

Active and passive surveillance form the backbone of New Zealand's early pest and disease detection system, and provide evidence about pest or disease status to support growing and maintaining export markets and trade for plant products. Effective surveillance increases the number of options available to emergency management teams to manage new biosecurity risks, from eradication and containment to pest management. An overview of 3 years of plant pest and disease surveillance data is presented, including an update of the surveillance system and potential areas for development. The importance of increasing awareness by Intermediaries (a group that includes scientists, crop scouts and biosecurity staff) of the Ministry for Primary Industries' exotic pest and disease hotline (0800 809966 ) to enable early risk management is highlighted.

\section{Accounting for imperfect detection when evaluating the effectiveness of invasive species control}

\author{
J.L. Moore ${ }^{1}$ and E. Gurarie ${ }^{2}$ \\ ${ }^{1}$ School of Biological Sciences, University of Monash, Victoria 3800, Australia \\ ${ }^{2}$ Department of Biology, University of Maryland, College Park, MD 20742, USA \\ Corresponding author: joslinm@unimelb.edu.au
}

Many invasive plant species are sparsely distributed across large areas. Management of these species is often undertaken using a search and destroy approach where people search the landscape and treat (destroy) any individuals found. However, detection is imperfect and so these searches need to be undertaken on multiple occasions. Given limited resources, an explicit objective is to optimize efforts by targeting those areas for follow up visits that have the highest predicted abundance. In order to simultaneously estimate both abundances and detection rates from data on search and destroy efforts, it is necessary to have a good model of the detection process itself. In a case study of invasive willow control across $120 \mathrm{~km}^{2}$ in alpine Australia, intensively monitored sample plots were used to characterise how detection rates depend on perceived abundance for three groups of willow control contractors. Bayesian models were used to fit an exponential detection function where the detection rate varied with plant size, between contractors, with the total number of willows treated, and on features of the contractors' movements. It was found that detection rates decreased with increasing abundance but areas with high abundance were subject to greater search effort. These models were combined with GPS tracking data representing 6 weeks of search and destroy missions to predict the remaining abundance of willows across the landscape, and hence areas that are priorities for follow up control were identified. 